MS24-O3 Diffuse X-ray scattering from ion-irradiated crystals: Monte Carlo simulations using multi-processing and GPU-accelerated computing

Jayanth Channagiri ${ }^{1}$, Alexandre Boulle ${ }^{1}$, Aurélien Debelle ${ }^{2}$

1. SPCTS - CNRS UMR 7315, Centre Européen de la Céramique, 12 rue atlantis, 87068 Limoges Cedex, France

2. CSNSM, Université Paris-Sud, CNRS-IN2P3, 91405 Orsay Cedex, France

email: jayanth.channagiri@etu.unilim.fr

Ion irradiation/implantation techniques are widely employed in various field of materials science, for instance, in the processing of semiconductor materials, the synthesis of nanostructures, to simulate radiative environments. In all cases, the interaction of energetic ions with matter creates defects in the target material along the path of the incoming ions, which in turn affect the properties of the material. In some particular cases, e.g. when the crystals contain homogeneously distributed defects of the same type and size, it is known since several decades that simple models for the coherent and diffuse scattering intensity can be obtained. However, real-world materials often contain heterogeneous distributionsof defects of different type, sizes, etc., which can only be analyzed using numerical methods that are often too resource-demanding (in terms of memory and CPU time) to be used on a regular basis.

In this work, we developed an innovative approach, where numerical crystals, containing up to $\sim 10^{9}$ unit cells, are computed and used to generate realistic two-dimensional diffuse scattering intensity distributions. The defect structures are generated within a Monte Carlo scheme using linear elasticity theory. High performance computing have been implemented using both multi-processing (i.e. distributing different computational tasks to the different cores of a multi-core central processing unit - CPU) and massively parallel computing on graphical processing units (GPUs). With this implementation, we were able to successfully compute, on a desktop workstation, reciprocal space maps (RSMs) from large crystals with an impressive speed-up of $\sim 190$ times when compared to the conventional single-processed computation (i.e. 5 minutes instead of $\sim$ 15 hours) [1]. The efficiency of the method will be illustrated with some selected examples, in particular, ion-irradiated yttria stabilized zirconia which exhibits a complex defect structure that significantly evolves with increasing radiation dose, leading to dramatically different RSMs [2].

[1] J. Channagiri et. al., J. Appl. Cryst. (2015) 48, 252-261.

[2] A. Debelle et. al., J. Appl. Phys. (2014) 115, 183504.

Keywords: Irradiated materials, Diffuse scattering, Parallel computing

\section{MS24-04 Fit of organic crystal structures to PDF Data}

Dragica Prill ${ }^{1}$, Martin U. Schmidt ${ }^{1}$, Pavol Juhás ${ }^{2}$, Simon J.L. Billinge $^{2,3}$

1. Goethe University, Frankfurt am Main, Germany

2. Brookhaven National Laboratory, Upton, USA

3. Columbia University, New York, USA

email: prill@chemie.uni-frankfurt.de

Local structures in crystalline, nanocrystalline and amorphous organic compounds can be investigated using pair distribution functions (PDF). The experimental determination of the PDF curves of organic compounds is similar to that of inorganic compounds. However, the fit of a structural model to a given PDF curve is at present much more challenging, because the molecular geometry (bond lengths, bond angles, torsion angles) has to be taken into account. Such a fit has rarely been done [1].

We have developed a method to perform fits of organic structures to experimental PDF data using the new Python-driven program package DiffPy-CMI [2]. Bond lengths, bond angles and torsion angles were kept fixed. The molecular position and the spatial orientation of the molecules were fitted together with lattice parameters, scale factor and isotropic displacement parameters [3]. Our approach using two different isotropic displacement parameters for intramolecular and intermolecular vibrations was applied during all calculations [4].

Three examples were chosen: crystalline samples of naphthalene and allopurinol, and a nanocrystalline sample of quinacridone. Synchrotron powder patterns of the three samples were recorded at NSLS (Brookhaven, USA) with $\lambda=0.18 \AA$. From these data the experimental PDF curves were derived with PDFgetX3 [5].

Crystal structural models of the three compounds were fitted to the experimental PDF curves. In all cases a good fit of the crystal structural models to the experimental PDF curves could be achieved. Furthermore, the fit was successful as well, when random values for the molecular position and orientation were chosen as starting points. Hence, the procedure also allows the solution of crystal structures of crystalline and nanocrystalline organic compounds by using the PDF curves [3].

[1] M.T. Dove, M.G. Tucker, D.A. Keen, Eur. J. Mineral. 2002, 14, 331-348

[2] C. L. Farrow, P. Juhás, S. J. L. Billinge, 2010, SrFit, unpublished.

[3] D. Prill, P. Juhás, S. J. L. Billinge, M. U. Schmidt, to be published.

[4] D. Prill, P. Juhás, M. U. Schmidt, S. J. L. Billinge, J. Appl. Cryst. 2015, 48, 171-178.

[5] P. Juhás, T. Davis, C. L. Farrow, S. J. L. Billinge, J. Appl. Cryst. 2013, 46, 560-566.

Keywords: Pair distribution function, PDF, organic compounds, nanocrystalline 